\title{
IMPACTO DO SOCO E DO CHUTE DOS ESPORTES DE COMBATE: UMA REVISÃO SISTEMÁTICA
}

\section{IMPACTO DEL PUÑETAZO Y DE LA PATADA EN LOS DEPORTES DE COMBATE: UNA REVISIÓN SISTEMÁTICA}

\section{PUNCH AND KICK IMPACT OF THE COMBAT SPORTS: A SYSTEMATIC REVIEW}

\author{
Nelson Kautzner Marques Junior \\ kautzner123456789junior@gmail.com \\ Universidade de Los Lagos, Santiago do Chile, Chile
}

Envio original: 2019-12-02 Reenviado: 2020-05-08, 2020-06-19 Aceitado: 2020-07-23

Publicado: 2020-07-30

Doi: https://doi.org/10.15517/pensarmov.v18i2.39894

\begin{abstract}
Resumo
O objetivo da revisão sistemática foi apresentar o impacto do soco e do chute de alguns esportes de combate. Os artigos foram identificados na internet durante setembro de 2018 a agosto de 2019. A coleta de artigos foi praticada no Google Scholar, no Research Gate e no PubMed. A revisão sistemática teve 19 artigos científicos. O maior impacto de soco do lutador masculino foi o direto do boxe com $4800 \pm 601$ newtons $(\mathrm{N})$. A lutadora do sexo feminino o maior impacto foi o jab do boxe com $1170,7 \pm 165,3 \mathrm{~N}$. O impacto do chute no taekwondo feminino (TKD) teve um resultado maior do que tui tchagui com $2490,3 \pm 846,2 \mathrm{~N}$ e no masculino teve um resultado de maior impacto do bandal tchagui do TKD de $6400 \pm 898 \mathrm{~N}$. Em conclusão, o impacto do soco e do chute dos esportes de combate é um conteúdo importante para o atleta conhecer o ataque mais eficaz.
\end{abstract}

Palavras-chave: esportes de combate, impacto do soco, impacto de chute, luta de competição.

\footnotetext{
Abstract

The objective of the systematic review was to present the punch and kick impact of some combat sports. The studies were identified in electronic databases from September 2018 to August 2019. Literature searches were conducted in Google Scholar, Research Gate, and PubMed. The systematic review had 19 scientific articles. The greater punch impact of the male fighter was of the boxing straight with $4800 \pm 601$ newtons $(\mathrm{N})$. Female fighter the greater
} 
punch impact was of the boxing jab with $1170.7 \pm 165.3 \mathrm{~N}$. The kick impact of the female taekwondo (TKD) had a greater result of the tui tchagui with $2490.3 \pm 846.2 \mathrm{~N}$ and in the male had a greater result of the TKD bandal tchagui impact of $6400 \pm 898 \mathrm{~N}$. In conclusion, the punch and kick impact of combat sports is an important content for the athlete to know the most effective attack.

Keywords: combat sports, punch impact, kick impact, competition fight.

\section{Resumen}

El objetivo de la revisión sistemática fue presentar el impacto del puñetazo y de la patada de algunos deportes de combate. Los artículos fueron recuperados de Internet durante septiembre de 2018 a agosto de 2019. La recopilación de los artículos se realizó por medio de Google Scholar, en Research Gate y en PubMed. La revisión sistemática contó con 19 artículos científicos. El mayor impacto de golpe del luchador masculino fue el recto del boxeo con $4800 \pm 601$ newtons $(\mathrm{N})$. La luchadora con el mayor impacto del puñetazo fue el jab del boxeo con $1170,7 \pm 165,3 \mathrm{~N}$. El impacto de la patada del taekwondo femenino (TKD) tuvo un mayor resultado realizando el tchagui con $2490,3 \pm 846,2 \mathrm{~N}$ y en el sexo masculino tuvo un mayor resultado del impacto realizando la bandal tchagui del TKD con $6400 \pm 898 \mathrm{~N}$. En conclusión, el impacto del puñetazo y de la patada de los deportes de combate es un contenido importante para que el atleta conozca el ataque más efectivo.

Palabras clave: deportes de combate, impacto del puñetazo, impacto de la patada, lucha de competencia.

\section{INTRODUÇÃO}

Aproximadamente em 5000 antes do aniversário de Cristo foi inciado a prática de artes marciais (Souza Junior, et al., 2015). As artes marciais como esportes de combate iniciaram na Grécia durante os Jogos Olímpicos da Antiguidade (Bishop, Bounty \& Devlin, 2013). A introdução dos esportes de combate nos Jogos Olímpicos modernos causou difusão dessas lutas pelo mundo (Franchini \& Del Vecchio, 2011).

A regra de vários esportes de combate é válido o nocaute. Então é importante o estudo do impacto do soco e do chute porque a melhor biomecânica do ataque causa um maior impacto (Venkatraman \& Nasiriavanaki, 2019). Portanto, o maior impacto do ataque tem mais chance de causar um nocaute porque a força do ataque é alto quando bate no oponente.

Quando o técnico estabelece o impacto do ataque é importante para monitorar a nível da força do soco e do chute durante a sessão (Sklyar, 2014). O impacto do ataque do lutador é útil para o treinador classificar o nível competitivo do atleta porque competidores de elite em 
geral tem maior impacto do ataque do que iniciantes e médios atletas (Estevan, Álvarez, Falco, Molina-García \& Castillo, 2011). Portanto, quando o técnico mensura o impacto do soco e do chute e de outros ataques, é possível de determinar a ação ofensiva com maior e menor impacto do ataque (Cynarski, Wasik, Szymczy \& Vences de Brito, 2018; Gavagan \& Sayers, 2017).

Entretanto, até a presente data, nenhuma revisão foi escrita sobre o impacto do soco e do chute dos esportes de combate (Fernandes, Wichi, Silva, Ladeira \& Ervilha, 2011; Hernández González, Sans-Rosell, Jové-Deltell, \& Reverter-Masia, 2018). Então, o objetivo da revisão sistemática foi apresentar o impacto do soco e do chute de alguns esportes de combate (taekwondô, karatê, boxe, boxe tailandês e yongmudo).

\section{METODOLOGIA}

O artigo seguiu a metodologia da revisão sistemática e meta-análise proposta pela PRISMA (Preferred Reporting Items for Systematic reviews and Meta-Analyses) (Moher, Liberati, Tetzlaff \& Altman, 2009). Os estudos foram identificados em bases de dados eletrônicas durante setembro de 2018 a agosto de 2019. A coleta dos artigos foi realizada no Google Acadêmico, no Research Gate e no PubMed. Nessas bases de dados eletrônicas foram consultadas usando as palavras chaves em inglês punch impact force e kick impact force.

Os artigos incluídos foram selecionados com as seguintes estratégias de pesquisa: (1) participantes (esportes de combate), (2) tipo de tarefa (impacto do soco e do chute) e (3) dados coletados (impacto em newton ou $\mathrm{N}$ com valor em número). Os artigos que não tiveram esses três critérios foram excluídos.

$\mathrm{Na}$ primeira fase, 4203 pesquisas foram encontradas com as palavras chaves informadas anteriormente. Então, na segunda fase, 102 artigos ficaram no estudo depois das duplicadas serem removidas - um total de 50 artigos excluídos. Os outros artigos excluídos na segunda fase tiveram um impacto em $\mathrm{N}$ somente com o gráfico (Halperin, Chapman, Martin, Lewthwaite \& Wulf, 2017; Markovic, Suzovic, Kasum \& Jaric, 2016), alguns artigos de revisão, outros artigos sobre a biomecânica do ataque, estudo do nocaute, o impacto do ataque do robô (Lee \& McGill, 2014) e outros temas sobre os esportes de combate que não foram foco da revisão sistemática - um total de 4051 artigos excluídos. Portanto, algumas duplicatas (50 artigos) e mais 4051 artigos deram um total de 4101 artigos excluídos - 4203 artigos foram encontrados - 4101 artigos excluídos = um total de 102 artigos permaneceram na revisão sistemática. Na terceira fase, 102 estudos o autor leu o título e o resumo de cada pesquisa e 34 artigos foram selecionados sobre o impacto do soco e do chute dos esportes de combate. Outras investigações foram excluídas, um total de 68 artigos, porque os estudos não mensuraram o impacto do ataque. 
Na quarta fase, o pesquisador leu 34 estudos durante 1 mês e o total de artigos reduziu para 19 estudos com chance de inclusão porque os artigos tinham resultado sobre o impacto do soco e do chute em $\mathrm{N}$. Os 15 artigos científicos foram excluídos dessa quarta fase por não apresentar no texto impacto do soco e do chute do ataque em $\mathrm{N}$ somente no gráfico e sem o valor. Também, o autor excluiu o impacto do ataque de outra técnica de ataque, por exemplo, Pinto Neto, Silva, Marzullo, Bolander e Bir (2013) verificou o impacto do ataque de mão aberta do kung fu. Desses estudos, 19 artigos foram incluídos na revisão sistemática (é a $5^{a}$ fase e é a última fase). Entretanto, 14 artigos de socos foram selecionados e 7 artigos de chutes foram selecionados, igual a 21 estudos. Mas isso ocorreu porque dois artigos científicos (Buśko, et al., 2016; Buśko \& Nikolaidis, 2018) foram sobre o impacto do soco e do chute. Então, o total da quantificação foi de 19 artigos científicos. Os detalhes da estratégia completa da coleta dos estudos foram listados em um diagrama de fluxo PRISMA, como mostrado na figura 1.

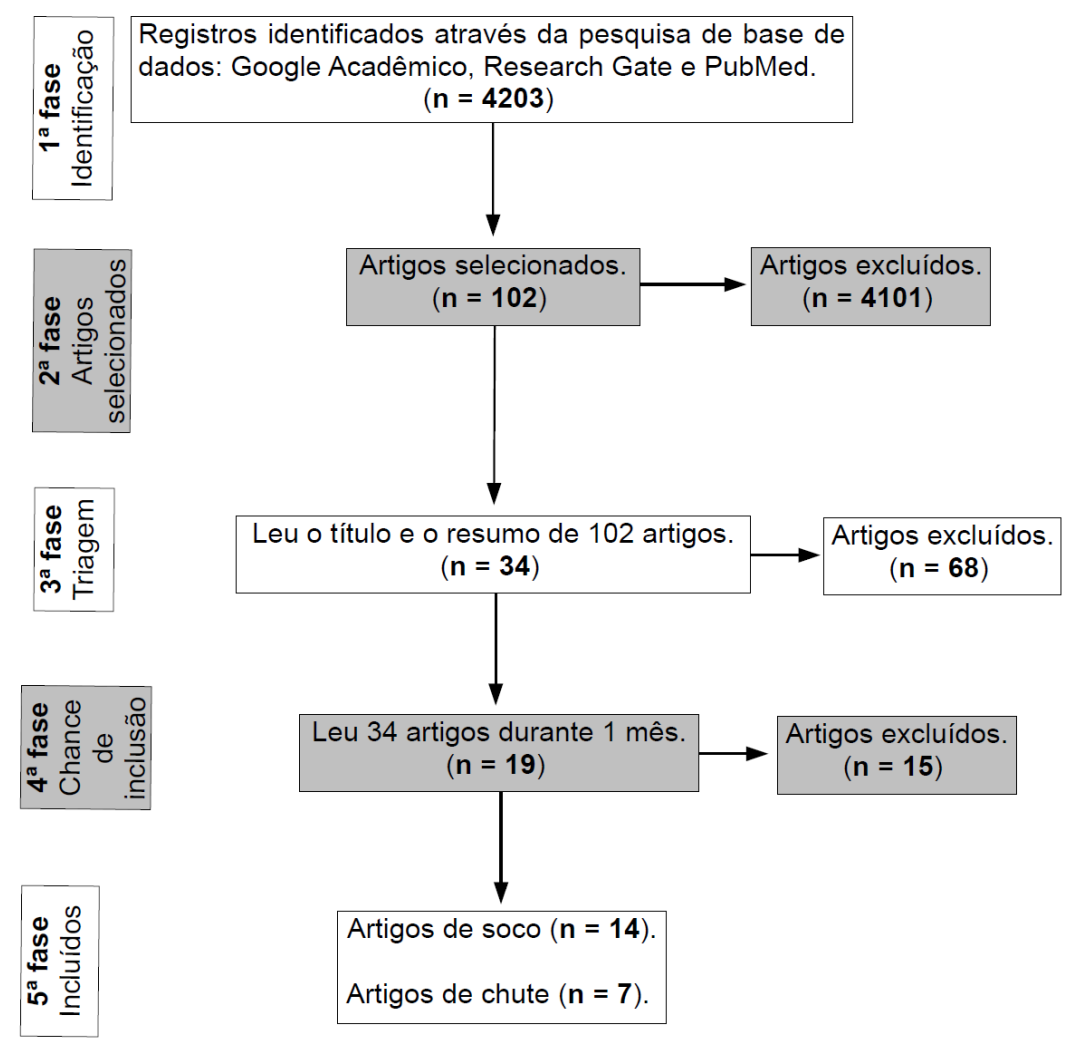

Figura 1. Fluxograma PRISMA da seleção dos artigos científicos. Fonte: elaborado pelo autor baseado em Moher et al. (2009).

O autor usou a escala de Galna, Peter, Murphy e Morris (2009) para a avaliação da qualidade dos estudos. Essa escala tem perguntas sobre o artigo e o pesquisador determinou o ponto (0 a 1) das 13 questões. A pontuação da escala de Galna et al. (2009) é estabelecida 
de 0 a 0,59 é baixo, 0,60 a 0,80 é médio e 0,81 a 1 é alto. Quando o artigo tem menos de 0,60 pontos conforme a avaliação do autor com a escala de Galna et al. (2009) foi considerado de baixa qualidade e foi excluído da revisão sistemática, mas isso não ocorreu. $A$ tabela 1 mostra como o autor analisou cada artigo.

O autor calculou o impacto relativo (IR) com uma divisão - IR = impacto do soco ou do chute em $\mathrm{N}$ : massa corporal total em $\mathrm{kg}=$ ? \%. Determinar o IR é importante porque isso possibilita de verificar o impacto do ataque do atleta de diferentes categorias de peso.

\section{RESULTADOS}

A avaliação dos artigos com a escala de Galna et al. (2009) foram estabelecidas de 12 artigos com alta qualidade e 8 estudos com média qualidade. A tabela 1 apresenta esses resultados. 
Tabela 1

Estudos avaliados com a escala de Galna et al. (2009)

\begin{tabular}{|c|c|c|c|c|c|c|c|c|c|c|c|c|c|c|}
\hline Estudo & 1 & 2 & 3 & 4 & 5 & 6 & 7 & 8 & 9 & 10 & 11 & 12 & 13 & $\begin{array}{l}\text { Média e Qualidade de } \\
\text { cada Estudo }\end{array}$ \\
\hline $\begin{array}{l}\text { Loturco, Nakamura, Artioli, Kobal, } \\
\text { Kitamura, Abad, Cruz, Romano, } \\
\text { Pereira e Franchini }(\underline{2016})\end{array}$ & 1 & 1 & 1 & 1 & 1 & 1 & 1 & 1 & 0 & 0 & 1 & 1 & 1 & 0,84 (alto) \\
\hline Walilko, Viano e Bir (2005) & 0,5 & 1 & 1 & 1 & 1 & 1 & 1 & 1 & 0 & 0 & 1 & 1 & 1 & 0,80 (médio) \\
\hline $\begin{array}{l}\text { Atha, Yeadon, Sandover e Parsons } \\
\qquad(\underline{1985})\end{array}$ & 1 & 1 & 1 & 1 & 1 & 1 & 1 & 1 & 0 & 0 & 1 & 1 & 1 & 0,84 (alto) \\
\hline Turner, Baker e Miller (2011) & 1 & 1 & 0,5 & 0,5 & 1 & 1 & 1 & 1 & 0 & 0 & 1 & 1 & 1 & 0,76 (médio) \\
\hline $\begin{array}{l}\text { Bingul, Bulgan, Tore, Aydin e Bal } \\
\qquad(\underline{2017})\end{array}$ & 1 & 1 & 1 & 1 & 1 & 1 & 1 & 1 & 0 & 0 & 1 & 1 & 1 & 0,84 (alto) \\
\hline Cruz, Nagata e Livramento (2014) & 1 & 1 & 1 & 0,5 & 1 & 1 & 1 & 1 & 0 & 0 & 1 & 1 & 1 & 0,80 (médio) \\
\hline Todeschini Viero (2012) & 1 & 1 & 1 & 0,5 & 1 & 1 & 1 & 1 & 0 & 0 & 1 & 1 & 1 & 0,80 (médio) \\
\hline Souza e Marques (ㅁ17) & 1 & 1 & 1 & 1 & 1 & 1 & 1 & 1 & 0 & 0 & 1 & 1 & 1 & 0,84 (alto) \\
\hline Touguinha (2014) & 1 & 1 & 1 & 1 & 1 & 1 & 1 & 1 & 0 & 0 & 1 & 1 & 1 & 0,84 (alto) \\
\hline Gulledge e Dapena (2008) & 1 & 1 & 1 & 0,5 & 1 & 1 & 1 & 1 & 0 & 0 & 1 & 1 & 1 & 0,80 (médio) \\
\hline $\begin{array}{c}\text { Dunn, Humberstone, Iredale e } \\
\text { Blazevich }(\underline{2019})\end{array}$ & 1 & 1 & 1 & 0,5 & 1 & 1 & 1 & 1 & 0 & 0 & 1 & 1 & 1 & 0,80 (médio) \\
\hline Cymarski et al. (2018) & 1 & 1 & 0,5 & 0,5 & 1 & 1 & 1 & 1 & 0 & 0 & 1 & 1 & 1 & 0,76 (médio) \\
\hline Estevan et al. (ㅁ11) & 1 & 1 & 1 & 0,5 & 1 & 1 & 1 & 1 & 0 & 1 & 1 & 1 & 1 & 0,88 (alto) \\
\hline Falco, Alvarez, Castillo, Estevan, & 1 & 1 & 1 & 1 & 1 & 1 & 1 & 1 & 0 & 1 & 1 & 1 & 1 & 0,92 (alto) \\
\hline
\end{tabular}


Mortos, Mugarra e Iradi (2009)

$\begin{array}{lllllllllllllll}\text { Gavagan e Sayers (2017) } & 1 & 1 & 1 & 1 & 1 & 1 & 1 & 1 & 0 & 1 & 1 & 1 & 1 & 0,92 \text { (alto) }\end{array}$

O’Sullivan, Chung, Lee, Kim, Kang, Kim e Shim (2009)

Buśko et al. (2016)

$1 \quad 1 \quad 11$

1

1

Venkatraman e Nasiriavanaki (2019)

Buśko e Nikolaidis (2018)

1

1

$1 \quad 1 \quad 1$

Fonte: elaborado pelo autor baseado em Galna et al. (2009) 
A numeração de 1 a 13 são as questões da Escala de Galna et al. (2009): 1. Objetivo do estudo ou questões estabelecidos claramente (Critério de Ponto: 1 - sim; 0,5 sim, falta detalhes ou clareza; 0 - não); 2. Detalhe dos participantes (número, idade, sexo, estatura, peso) (Critério de Ponto: 0 a 1); 3. Descrição da seleção da amostra (Critério de Ponto: 1 - sim; 0,5 - sim, falta detalhes ou clareza; 0 - não); 4. Detalhe dos critérios de inclusão e exclusão (1 - sim; 0,5 - sim, falta detalhes ou clareza; 0 - não); 5. Covariáveis controladas (0 a 1); 6. Resultados principais claramente descritos (1 - sim; 0,5 - sim, falta detalhes ou clareza; 0 - não); 7. Metodologia adequada para o estudo ser reproduzido (amostragem dos participantes, equipamento, procedimento, processamento dos dados, estatística) (0 a 1); 8. Metodologia capaz de responder as questões do estudo (amostragem dos participantes, equipamento, procedimento, processamento dos dados, estatística) (1 sim; 0 - não); 9. Confiabilidade da metodologia foi determinada (1 - sim; 0 - não); 10. Validade interna da metodologia foi determinada (1 - sim; 0 - não); 11. Questões da pesquisa foram respondidas adequadamente na discussão (1 - sim; 0 - não); 12. As principais descobertas foram apoiadas nos resultados (1 - sim; 0 - não); 13. Os principais resultados foram interpretados de uma maneira lógica e apoiados na literatura (1 - sim; 0 - não). Qualidade dos Estudos: 0 a 0,59 é baixo, 0,60 a 0,80 é médio e 0,81 a 1 é alto.

A tabela 2 resumo dos 19 estudos selecionados. 
Tabela 2

Delineamento dos estudos da revisão sistemática

\begin{tabular}{|c|c|c|c|}
\hline ESTUDO & AMOSTRA & PROCEDIMENTOS & RESULTADOS \\
\hline $\begin{array}{l}\text { Bingul et al. } \\
\quad(\underline{2017})\end{array}$ & $\begin{array}{l}\text { Boxeadores meio leve de } 19,33 \pm 2,11 \\
\text { anos da seleção da Turquia }(n=9) \text {. }\end{array}$ & $\begin{array}{l}\text { Os atletas fizeram um aquecimento de } 10 \text { minutos } \\
\text { (min) e depois realizaram um soco com a mão } \\
\text { direita no saco de pancada na posição tradicional } \\
\text { southpaw e o impacto foi coletado em } 3 D \text { e por } \\
\text { cálculos matemáticos. }\end{array}$ & Detalhes veja os resultados no texto. \\
\hline $\begin{array}{l}\text { Walilko et al. } \\
\qquad(\underline{2005})\end{array}$ & $\begin{array}{l}\text { Boxeadores olímpicos ( } \mathrm{n}=10 \text {, peso } \\
\text { mosca de } 50,80 \mathrm{~kg} \text {, peso meio leve } \\
\text { de } 63,05 \mathrm{~kg} \text {, peso médio de } 74,84 \mathrm{~kg} \\
\text { e peso super pesado de } 108.86 \mathrm{~kg} \text { ). }\end{array}$ & $\begin{array}{l}\text { Boxeadores realizaram } 30 \text { socos na face do boneco } \\
\text { que coletou o impacto do soco através do Seven } \\
\text { Endevco (San Juan Capistrano, CA). }\end{array}$ & Detalhes veja os resultados no texto e na figura $2 \mathrm{~B}$. \\
\hline $\begin{array}{l}\text { Atha et al. } \\
\text { (1985) }\end{array}$ & $\begin{array}{l}\text { Lutador de boxe Frank Bruno com } \\
\text { classificação mundial no peso } \\
\text { pesado. }\end{array}$ & $\begin{array}{l}\text { Bruno praticou um aquecimento com } 7 \text { socos e } \\
\text { depois realizou o mesmo soco no acelerômetro que } \\
\text { mensurou o impacto do soco. }\end{array}$ & Detalhes veja os resultados no texto. \\
\hline $\begin{array}{l}\text { Turner et al. } \\
\qquad \underline{(\underline{2011})}\end{array}$ & $\begin{array}{l}\text { Boxeadores ingleses de } 23,1 \text { a } 23,6 \\
\text { anos. Alguns eram lutadores de elite } \\
(\mathrm{n}=7,69,9 \pm 8,6 \mathrm{~kg}) \text {, intermediários }(\mathrm{n} \\
=8,73,4 \pm 8,2 \mathrm{~kg}) \text { e iniciantes }(\mathrm{n}=8, \\
78,5 \pm 8,9 \mathrm{~kg}) .\end{array}$ & $\begin{array}{l}\text { Boxeadores praticaram o jab e o direto sobre o } \\
\text { dinamômetro que determinou o impacto. }\end{array}$ & $\begin{array}{l}\text { Lutador de boxe de elite (direto com } 4800 \pm 601 \mathrm{~N} \text { e jab } \\
\text { com } 2847 \pm 596 \mathrm{~N} \text { ), intermediário (direto com } 3722 \pm 375 \\
\mathrm{~N} \text { e jab com } 2283 \pm 355 \mathrm{~N} \text { ) e iniciante (direto com } \\
2381 \pm 328 \mathrm{~N} \text { e jab com } 1604 \pm 273 \mathrm{~N})(p>0,05) \text {. }\end{array}$ \\
\hline $\begin{array}{l}\text { Loturco et al. } \\
\qquad(\underline{2016})\end{array}$ & $\begin{array}{l}\text { Boxeadores da seleção brasileira } \\
\text { amador ( }=9 \text { de homens, } \mathrm{n}=6 \text { de } \\
\text { mulheres) de } 25,9 \pm 4,7 \text { anos do peso } \\
\text { mosca ao peso pesado, ambos } \\
\text { tinham massa corporal de } \\
64,56 \pm 12,1 \mathrm{~kg} .\end{array}$ & $\begin{array}{l}\text { O lutador socou a plataforma de força Bad Boy } \\
\text { (San Diego, CA) que foi fixada na parede com } 12 \\
\text { socos que foram } 3 \text { socos de cada tipo de soco (jab } \\
\text { e cruzado na posição padrão, jab e cruzado na } \\
\text { posição escolhida). Depois de } 3 \text { socos, o lutador } \\
\text { descansou por } 15 \text { a } 60 \text { segundos para a fadiga não } \\
\text { interferir no teste. Durante cada soco, o lutador de } \\
\text { boxe foi verbalmente motivado pelos pesquisadores } \\
\text { a praticar o soco com máximo de força. }\end{array}$ & 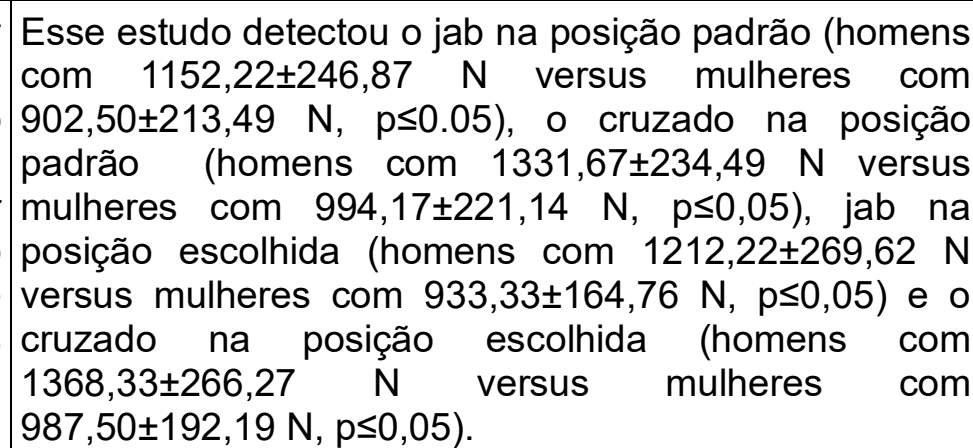 \\
\hline
\end{tabular}


Buśko et al. Boxeadores (mulheres: $n=7,17 \pm 1,1$ Os lutadores de boxe socaram no saco de pancada $\mathrm{O}$ impacto do soco direto (homens: $1102,9 \pm 430,7 \mathrm{~N}$, (2016) anos e $68,9 \pm 12,4 \mathrm{~kg}$, homens: $\mathrm{n}=13$, e o dinamômetro coletou o impacto.

$17,1 \pm 1,5$ anos e $68,7 \pm 12 \mathrm{~kg}$ ) mulheres: $848,4 \pm 218,5 \mathrm{~N}$ ) e o impacto do jab (homens: $1592,5 \pm 507,1 \mathrm{~N}$, mulheres: $1170,7 \pm 165,3 \mathrm{~N}$ ) tiveram diferença estatística somente no mesmo impacto entre homens versus mulheres $(p \leq 0,05)$.

Dunn et al. Boxeadores da Austrália ( $n=15$, Boxeadores praticaram um aquecimento de 5 min e Impacto do jab foi de $841 \pm 180 \mathrm{~N}$, cruzado foi de (2019) $\quad 17,5 \pm 0,5$ anos, $73 \pm 14 \mathrm{~kg}$ depois praticaram o teste de soco por 3 minutos. $1818 \pm 33 \mathrm{~N}$, cruzado com o braço da frente da guarda Os lutadores realizaram 6 repetições do soco no foi de $2481 \pm 428 \mathrm{~N}$ e o cruzado com o braço de trás da ciclo de 30 segundos. Em cada ciclo, os atletas guarda foi de $2622 \pm 288 \mathrm{~N}(p>0,05)$. praticaram 5 socos na plataforma de força combinado com um sinal visual e auditivo por 5 segundos e depois ocorreu uma pausa de 5 segundos. Durante cada ciclo, um tipo de soco combinado foi realizado $\left(1^{a}\right.$ combinação: jab, cruzado, jab, cruzado e cruzado, $2^{\mathrm{a}}$ combinação: 3 cruzados com o braço da frente da guarda, $3^{a}$ combinação: 3 cruzados com o braço de trás da guarda). O mesmo teste do soco foi realizado em outro dia para verificar a confiabilidade da tarefa.

Touguinha Homens de MMA com 18 a 30 anos Antes do estímulo visual, o lutador praticou um $\mathrm{O}$ impacto do soco direto dos profissionais do MMA foi (2014) ( $n=48)$, sendo que 6 eram soco no saco de pancada e o impacto foi de $2894,83 \pm 421,51 \mathrm{~N}$ e dos amadores do MMA foi de profissionais $(87,60 \mathrm{~kg})$ e 42 eram mensurado pela análise cinemática e por cálculos $2068,88 \pm 626,7 \mathrm{~N}(\mathrm{p}>0,05)$. amadores $(82,73 \mathrm{~kg})$. matemáticos.

Todeschini Karatecas faixa pretas $1^{\circ}$ dan do Os karatecas realizaram o gyaku zuki na zenkutsu O impacto do gyaku zuki foi $3501,93 \pm 12,28 \mathrm{~N}$ e do

Viero (2012) estilo shotokan do sexo masculino dachi e praticaram o choku zuki. Ambos os socos choku zuki foi de $1421,96 \pm 156,69$ a $2850,72 \pm 362,04 \mathrm{~N}$. $(n=9,85,88 \pm 16,31 \mathrm{~kg})$. foram realizados na plataforma de força AMTI, O impacto do soco foi convertido para porcentagem modelo OR6-GT. conforme a massa corporal. Grupo $1(n=2)$ o gyaku zuki na zenkutsu dachi com alta média do peso em percentual (média de 111,29\%) teve uma média do soco de $2.97 \%$. Mas o grupo $2(n=7)$ o gyaku zuki na zenkutsu dachi com menor média do peso em percentual (média de $82,27 \%$ ) teve uma média do soco de $3,30 \%$. O teste "t" detectou estatística diferença do impacto do gyaku zuki na zenkutsu dachi, $t(8)=4,15, p$ 


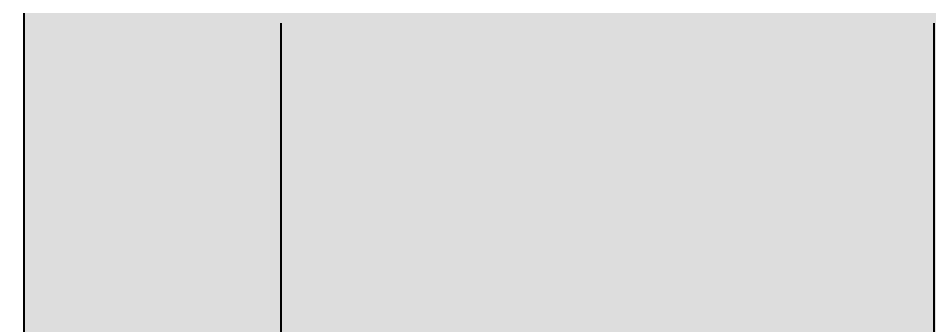

Cruz et al. Karatecas adultos $(n=11)$

$(\underline{2014})$

Karatecas praticaram o gyaku zuki durante 3 séries Impacto do gyaku zuki foi de 700 a $1400 \mathrm{~N}$. O estudo e 4 repetições com um intervalo de 4 segundos não usou a estatística inferencial para análise.

(seg). Os socos foram na plataforma rígida de

$35 \times 35 \mathrm{~cm}$ emborrachado com placa rígida de 200

$\mathrm{kg}$ com célula de carga, tendo um condicionador

HBM Spider 8 e um programa chamado Catman,

um computador e uma câmera digital.

Souza e

Karatecas faixa pretas do estilo Karatecas praticaram com o braço dominante o

Marques shotokan (n=8, 20,25 $\pm 4,13$ anos,

$(\underline{2017})$ $72,41 \pm 9,62 \mathrm{~kg})$.

$=0,003$. Grupo $1(n=5)$ no choku zuki com alta média do peso em percentual (média de $85,41 \%$ ) teve uma média do soco de $2.58 \%$. Mas o grupo $2(n=4)$ no choku zuki com menor média do peso em percentual (média de 109,72\%) teve uma média do soco de $3,82 \%$. O teste "t" não detectou diferença estatística do impacto do choku zuki, $\mathrm{t}(8)=-1,18, \mathrm{p}=0,27$.
Venkatraman Karateca com 7 anos de prática do e Nasiriavanaki (2019)

Buśko e

Nikolaidis (2018)

Lutadores de taekwondô (TKD) da
WTF da seleção da Polônia $(n=6$, $17,7 \pm 0,7$ anos e $62,3 \pm 6 \mathrm{~kg}$ ).

\begin{tabular}{c|l} 
& \\
$\begin{array}{c}\text { Gulledge e } \\
\begin{array}{c}\text { Dapena } \\
(\underline{2008)})\end{array}\end{array}$ & $\begin{array}{l}\text { Lutadores de esportes de combate } \\
(\mathrm{n}=6 \text { do kung fu, } \mathrm{n}=3 \text { do karatê e } n=3\end{array}$ \\
do TKD).
\end{tabular}
(2008) do TKD). gyaku zuki no makiwara e foi coletado o impacto
através do strain gauge que passou os dados para
um instrumento eletrônico.
O impacto do gyaku zuki foi de $1567,44 \pm 164,74$ a $2218,19 \pm 71,93$ N. O estudo detectou diferença estatística da idade versus o impacto do soco $(p \leq 0,05)$, da experiência prática no makiwara versus o impacto do soco $(p \leq 0,01)$. O impacto foi uma variável dependente.

O karateca praticou 8 vezes o gyaku zuki na O impacto do gyaku zuki foi de $300,4 \pm 95,52 \mathrm{~N}$ e o circular plataforma de força e o sensor enviou os gyaku zuki sem rotação do tronco foi de $272,4 \pm 74,12 \mathrm{~N}$. dados para o sistema que detectou o valor do Mas esses dados não foram comparados pela impacto. estatística inferencial.

Os lutadores de TKD praticaram um aquecimento e Impacto do baro jirugui foi de 1659,2 $\pm 254,2 \mathrm{~N}$ e do depois eles efetuaram 3 socos de cada tipo de jirugui cruzado com o braço de frente da guarda foi de técnica (baro jirugui que é similar ao gyaku zuki e o $1843,8 \pm 453,3 \mathrm{~N}$. O impacto do baro jirugui versus o jirugui cruzado com o braço de frente da guarda) no jirugui cruzado não teve diferença estatística $(p>0,05)$. saco de pancada que teve um dinamômetro que mensurou o impacto. karatê. O soco foi praticado na plataforma de força de $790 \pm 13 \mathrm{~N}$. O impacto do soco teve diferença 


\begin{tabular}{|c|c|c|c|}
\hline & & $\begin{array}{l}\text { AMTIOR6-7 que detectou o sinal do impacto e } \\
\text { encaminhou para o computador. }\end{array}$ & estatística $(p \leq 0,001)$ \\
\hline $\begin{array}{l}\text { Buśko et al. } \\
\qquad \underline{(2016})\end{array}$ & 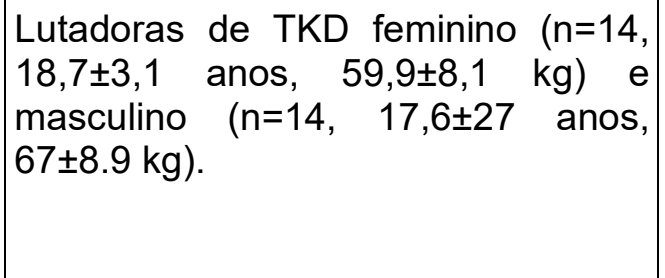 & $\begin{array}{l}\text { Lutadores de TKD chutaram o saco de pancada e o } \\
\text { dinamômetro coletou o impacto. Os lutadores } \\
\text { praticaram o tui tchagui, o bandal tchagui com a } \\
\text { perna da frente e o bandal tchagui com a perna de } \\
\text { trás. }\end{array}$ & $\begin{array}{l}\text { O impacto do tui tchagui foi de } 3426,1 \pm 911 \mathrm{~N} \text {, bandal } \\
\text { tchagui com a perna da frente foi de } 1206,7 \pm 239,5 \mathrm{~N} \text { e } \\
\text { com a perna de trás foi de } 2072,3 \pm 472 \mathrm{~N} \text {. O estudo } \\
\text { detectou diferença estatística entre o bandal tchagui da } \\
\text { perna da frente versus da perna de trás ( } p \leq 0,05 \text { ). } \\
\text { Detalhes veja os resultados no texto e na figura } 3 \text { e } 5 \text {. }\end{array}$ \\
\hline $\begin{array}{l}\text { Gavagan e } \\
\text { Sayers } \\
(\underline{2017})\end{array}$ & $\begin{array}{l}\text { Lutadores de boxe tailandês }(\mathrm{n}=8, \\
22,3 \pm 4,1 \text { anos, } 1,74 \pm 0,09 \mathrm{~m} \text { e } \\
65,6 \pm 8,4 \mathrm{~kg}), \text { de karatê }(\mathrm{n}=8, \\
30,3 \pm 10,7 \text { anos, } 1,78 \pm 0,13 \mathrm{~m} \text { e } \\
84.5 \pm 20.1 \mathrm{~kg}) \text { e de TKD }(\mathrm{n}=8 \\
28,6 \pm 9,5 \text { anos, } 1,77 \pm 0,05 \mathrm{~m} \text { e } \\
95,8 \pm 13,4 \mathrm{~kg}) \text { mestre em esportes de } \\
\text { combate. }\end{array}$ & $\begin{array}{l}\text { Os lutadores aqueceram por } 10 \text { min e depois } \\
\text { realizaram o chute semicircular por } 10 \text { seg. O } \\
\text { lutador boxe tailandês praticou o chute lateral, o } \\
\text { karateca realizou o mawashi geri e o lutador de } \\
\text { TKD fez o bandal tchagui. Depois de cada chute, o } \\
\text { lutador praticou uma pausa de } 60 \text { seg. O chute foi } \\
\text { praticado no alvo tendo o strain gauge que coletou } \\
\text { o impacto e os dados foram transmitidos para o } \\
\text { computador. }\end{array}$ & Detalhes veja os resultados no texto e na figura 5. \\
\hline $\begin{array}{l}\text { Cynarski et } \\
\text { al. }(\underline{\underline{2018}})\end{array}$ & $\begin{array}{l}\text { Karatecas do } 2^{\circ} \text { dan da faixa preta } \\
\text { do estilo Idokan }(n=1,36 \text { anos e } 97 \\
\mathrm{kg}) \text {. }\end{array}$ & $\begin{array}{l}\text { O karateca na zenkutsu dachi praticou o mae geri } \\
\text { na plataforma de força Kistler do tipo } 9286 \text { AA. }\end{array}$ & $\begin{array}{l}\text { O karateca praticou o mae geri (é o chute frontal) com a } \\
\text { perna esquerda e o impacto foi de } 1082,42 \mathrm{~N} \text { e com a } \\
\text { perna direita foi de } 1208,69 \mathrm{~N} \text {. Mas o autor não fez a } \\
\text { estatística inferencial. }\end{array}$ \\
\hline $\begin{array}{l}\text { Buśko e } \\
\text { Nikolaidis } \\
(\underline{2018)}\end{array}$ & $\begin{array}{l}\text { Lutadores de TKD da WTF da } \\
\text { seleção da Polônia }(n=6,17,7 \pm 0,7 \\
\text { anos e } 62,3 \pm 6 \mathrm{~kg}) \text {. }\end{array}$ & $\begin{array}{l}\text { Os lutadores de TKD praticaram um aquecimento e } \\
\text { depois eles fizeram os chutes no saco de pancada } \\
\text { que teve um dinamômetro que mensurou o } \\
\text { impacto. }\end{array}$ & $\begin{array}{l}\text { O impacto do tui tchagui foi de } 3568 \pm 1306 \mathrm{~N} \text {, do bandal } \\
\text { tchagui com a perna da frente foi de } 3409,5 \pm 581,3 \mathrm{~N} \text { e } \\
\text { com a perna de trás foi de } 4580,8 \pm 641,1 \mathrm{~N} \text {. O estudo } \\
\text { detectou diferença estatística entre o bandal tchagui } \\
\text { com a perna da frente versus com a perna de trás } \\
(p \leq 0,05) \text {. }\end{array}$ \\
\hline $\begin{array}{l}\text { O`Sullivan et } \\
\text { al. }(\underline{\underline{2009}})\end{array}$ & $\begin{array}{l}\text { Yongmudo }(n=5,71,5 \pm 6,5 \mathrm{~kg}) \text { e TKD } \\
(\mathrm{n}=5,66,9 \pm 6,1 \mathrm{~kg}) \text { praticantes por } 10 \\
\text { anos esses esportes. }\end{array}$ & $\begin{array}{l}\text { Os lutadores aqueceram e depois fizeram o bandal } \\
\text { tchagui no tronco e no rosto do alvo. O impacto foi } \\
\text { coletado por um acelerômetro. }\end{array}$ & $\begin{array}{l}\text { O impacto do bandal tchagui do yongmudo foi de } \\
6393 \pm 1382 \mathrm{~N} \text { no tronco e } 5475 \pm 1293 \mathrm{~N} \text { no rosto. } \\
\text { impacto do bandal tchagui do TKD foi de } 6400 \pm 898 \mathrm{~N} \\
\text { no tronco e } 5419 \pm 659 \mathrm{~N} \text { no rosto. O estudo detectou } \\
\text { diferença estatística ( } \leq 0,05 \text { ) do impacto do bandal } \\
\text { tchagui no tronco (yongmudo versus TKD) e no rosto }\end{array}$ \\
\hline
\end{tabular}


O impacto do soco do lutador de boxe masculino sem informação da técnica efetuada teve três estudos. Bingul, Bulgan, Tore, Aydin e Bal (2017) detectou durante a posição southpaw $(1987,42 \pm 341,95 \mathrm{~N})$ versus a posição tradicional $(1616,96 \pm 432,92 \mathrm{~N})$ uma diferença estatística no impacto do soco $(p \leq 0,05)$. Walilko, Viano e Bir (2005) evidenciou diferença estatística $(p \leq 0,02)$ em várias comparações das categorias por peso, detalhes na figura 2B. Essas categorias por peso tiveram um impacto do soco entre 1990 a $4741 \mathrm{~N}$. Atha, Yeadon, Sandover e Parsons (1985) identificaram $4096 \mathrm{~N}$ do impacto do soco do lutador de boxe profissional Frank Bruno e $6320 \mathrm{~N}$ foi a força estimada do soco desse lutador na cabeça de um ser humano. A figura 2A apresenta o mínimo e máximo do impacto do soco do boxe e a figura 2B mostra o impacto do soco de quatro categorias por peso.

A

Impacto do Soco do Boxe Masculino
(mínimo e máximo)

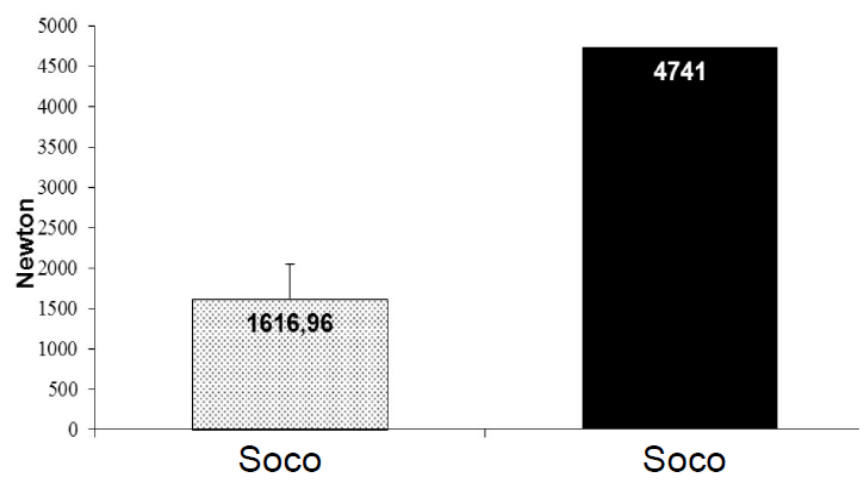

B

Impacto do Soco do Boxe Masculino (categoria por peso)

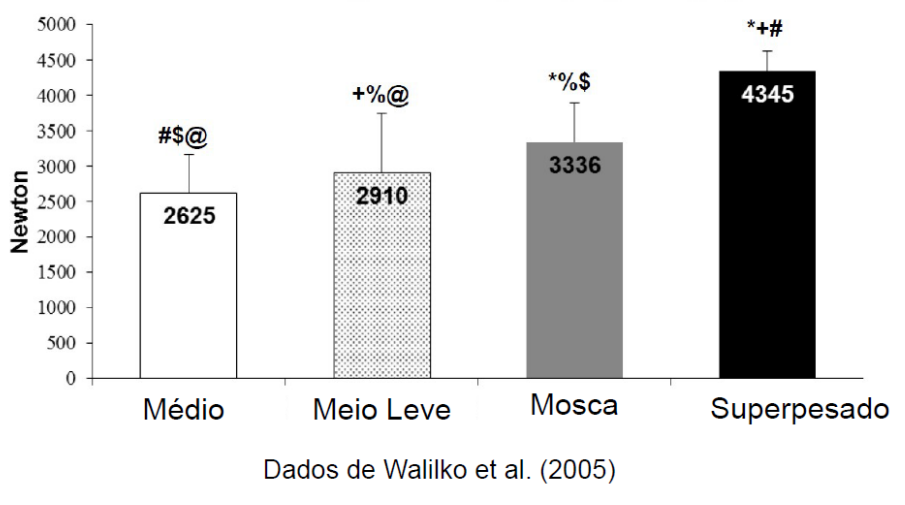

Diferença estatística $(p \leq 0,02)$ : superpesado versus mosca*, superpesado versus meio leve+, superpesado versus médio\#, mosca versus meio leve $\%$, mosca versus médio\$ e meio leve versus médio@.

Figura 2. (A) Impacto do soco do lutador de boxe. (B) impacto do soco do boxe por categoria de peso. Fonte: ilustrações elaboradas pelo autor baseado nos dados de (Atha, et al., 1985; Bingul, et al., 2017; Walilko, et al., 2005).

O impacto do soco com a técnica mencionada teve 11 estudos, detalhes na tabela 2. O tipo de impacto do soco teve um total de 5 estudos de boxe (Buśko, et al., 2016; Dunn, Humberstone, Iredale \& Blazevich, 2019; Loturcoet al., 2016; Touguinho, 2014; Turner, Baker \& Miller, 2011), 4 artigos de karatê (Cruz, Nagata \& Livramento, 2014; Souza \& Marques, 2017; Venkatraman \& Nasiriavanaki, 2019; Todeschini Viero, 2012), 1 estudo de taekwondô (Buśko \& Nikolaidis, 2018) e 1 artigo com vários esportes de combate (Gulledge \& Dapena, 2008). Os karatecas fizeram dois tipos de socos, o gyaku zuki é um soco com rotação do tronco com a mão de trás da guarda com o lutador na base zenkutsu dachi. Os lutadores de taekwondô efetuaram duas técnicas de soco, o baro jirugui é um soco com rotação do tronco 
com a mão de trás da guarda e o jirugui cruzado é um soco semicircular com o braço da frente da guarda. A figura $3 \mathrm{~A}$ é o máximo impacto do soco masculino e a $\underline{3} \mathrm{~B}$ é o máximo impacto do soco feminino. Esses valores são a média mais elevada do impacto do soco de cada esporte de combate.

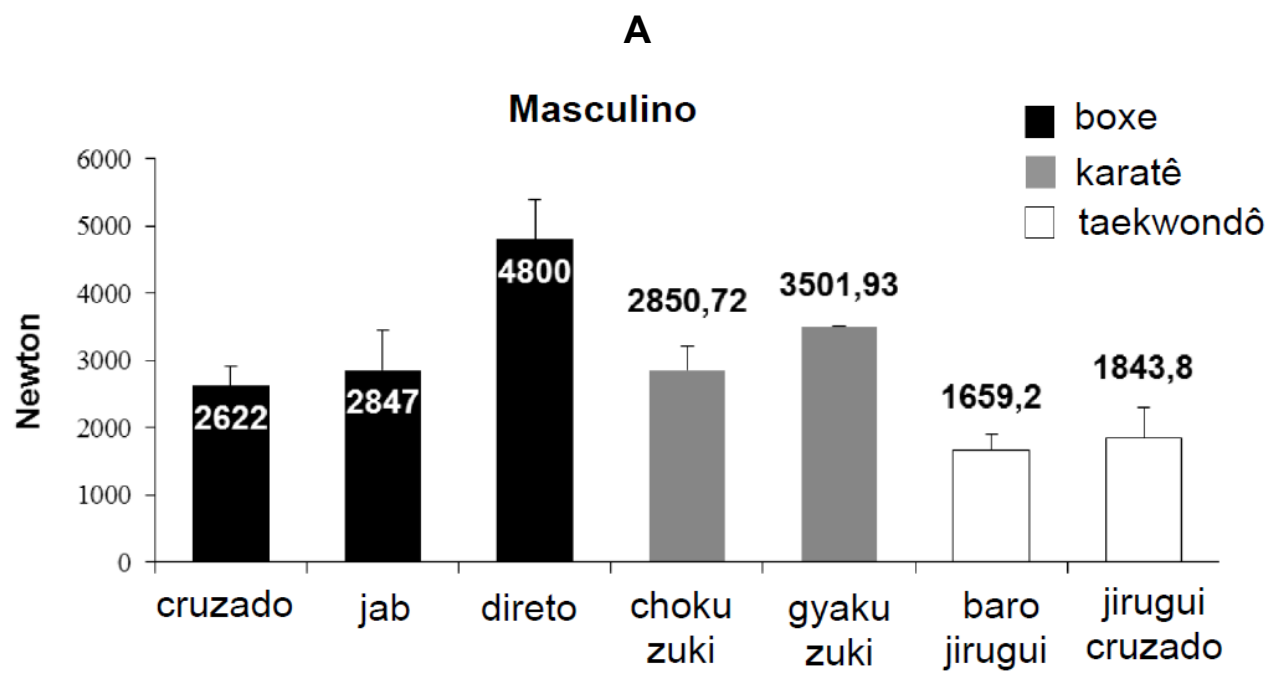

B

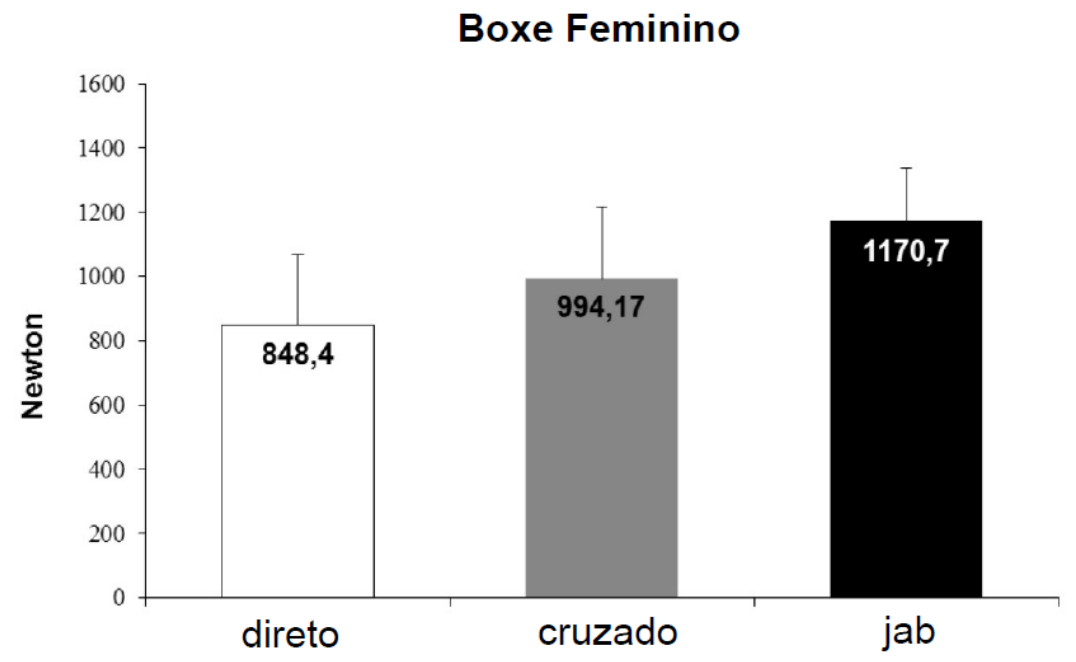

Figura 3. Impacto do soco (A) masculino. B) feminino conforme a técnica. Fonte: ilustrações elaboradas pelo autor baseado nos dados de (Buśko et al., 2016; Buśko \& Nikolaidis, 2018; Cruz et al., 2014; Dunn et al., 2019; Gulledge \& Dapena, 2008; Loturco et al., 2016; Souza \& Marques, 2017; Todeschini Viero, 2012; Touguinho, 2014; Turner et al., 2011; Venkatraman \& Nasiriavanaki, 2019).

O impacto do chute foi estudado em três técnicas, o chute frontal (mae geri), o chute semicircular (bandal tchagui, mawashi geri, e chute lateral) e o chute de costas (tui tchagui). 
O mae geri no artigo o karateca praticou o chute frontal com a perna de trás da base. $O$ bandal tchagui do taekwondô (TKD), o mawashi geri do karatê e o chute lateral do boxe tailandês são chutes semicirculares com a perna de trás da base ou com a perna da frente da base. O tui tchagui o lutador pratica um giro de $180^{\circ}$ e depois faz o chute de costas olhando por cima do ombro.

Somente a investigação de Buśko et al. (2016) estudou o impacto do chute feminino. Os pesquisadores mensuraram das lutadoras de TKD o impacto do tui tchagui, o impacto do bandal tchagui com a perna da frente da base e o impacto do bandal tchagui com a perna de trás da base. O estudo detectou diferença estatística do bandal tchagui com a perna da frente da base versus o bandal tchagui com a perna de trás da base $(p \leq 0,001)$, tendo tamanho do efeito alto de 0,81 . A figura 4 ilustra esse resultado.

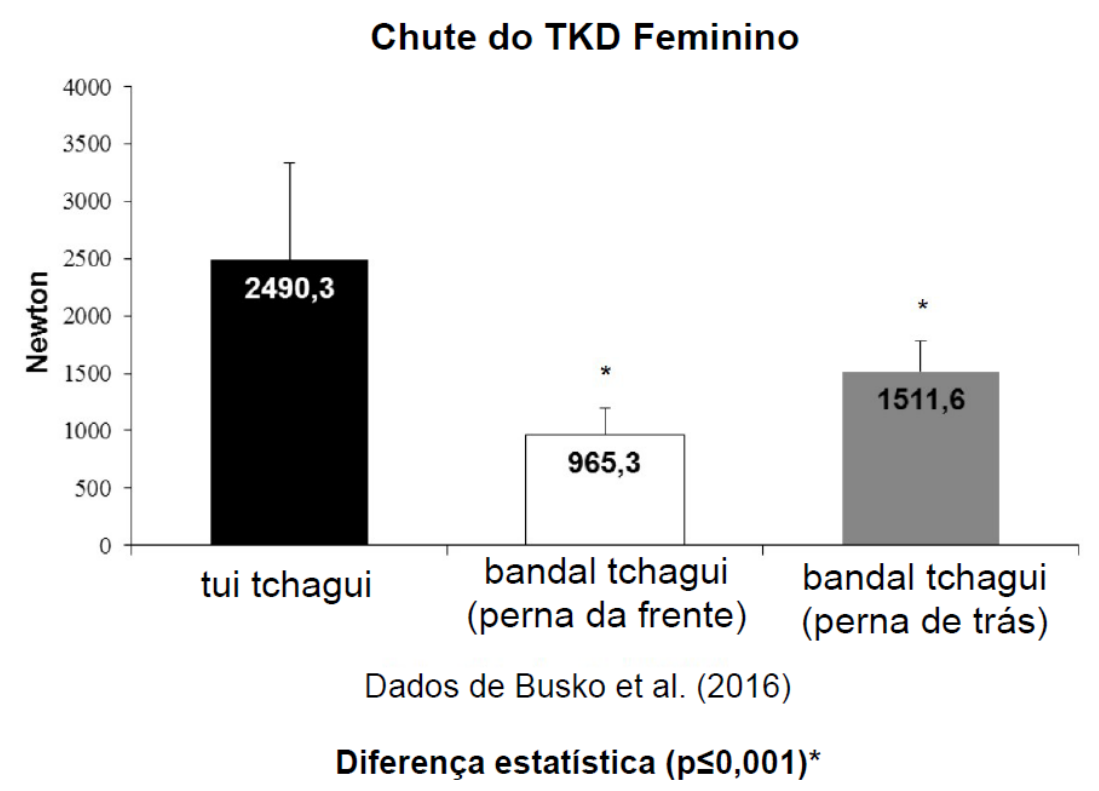

Figura 4. Impacto do chute feminino do TKD. Fonte: ilustração elaborada pelo autor baseado nos dados de Buśko et al. (2016).

Os outros estudos sobre o chute foram sobre o lutador masculino. Gavagan e Sayers (2017) determinaram o impacto do chute semicircular de três esportes de combate (boxe tailandês praticou o chute lateral, karatê praticou mawashi geri e TKD praticou o bandal tchagui). O resultado do estudo foi conforme a especificidade da luta porque o TKD teve maior impacto do chute semicircular, o chute é prioridade nesse esporte de combate. Entretanto, essas comparações entre cada chute semicircular conforme a luta não tiveram diferença estatística $(p>0,28)$. A figura 5 apresenta esse resultado. 


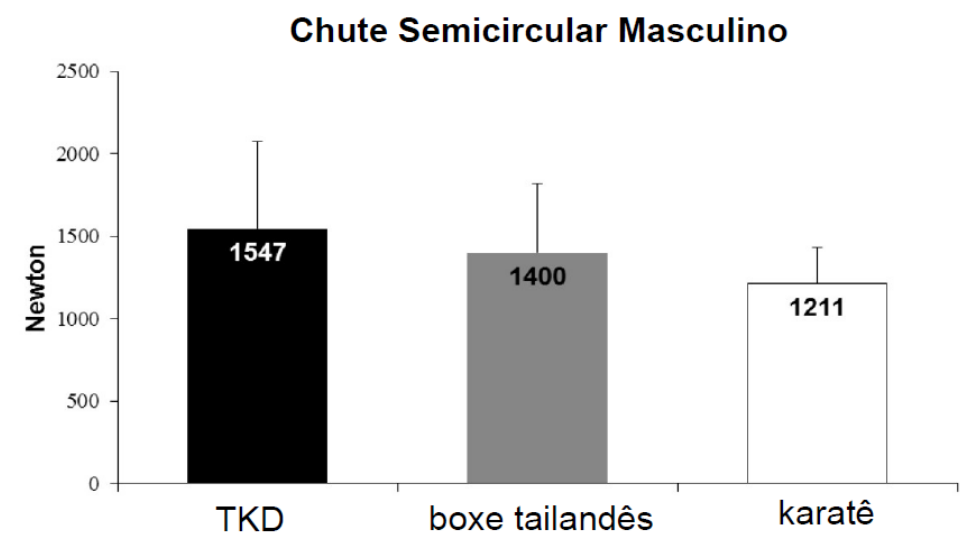

Figura 5. Impacto do chute semicircular conforme o esporte de combate. Fonte: ilustração elaborada pelo autor baseado nos dados de Gavagan e Sayers (2017).

O impacto do chute com a técnica mencionada foi de 7 estudos, detalhes na tabela 2 . 0 tipo de impacto do chute teve um total de 1 estudo do karatê (Cynarski et al., 2018), 1 artigo de três lutas (boxe tailandês, karatê e TKD) (Gavagan \& Sayers, 2017), 1 estudo de duas lutas (TKD e yongmudo) (O Sullivan et al., 2009) e 4 artigos do TKD (Buśko et al., 2016; Buśko \& Nikolaidis, 2018; Estevan et al., 2011; Falco et al., 2009). Os resultados da figura 6 são a média mais elevada do impacto do chute de cada esporte de combate.

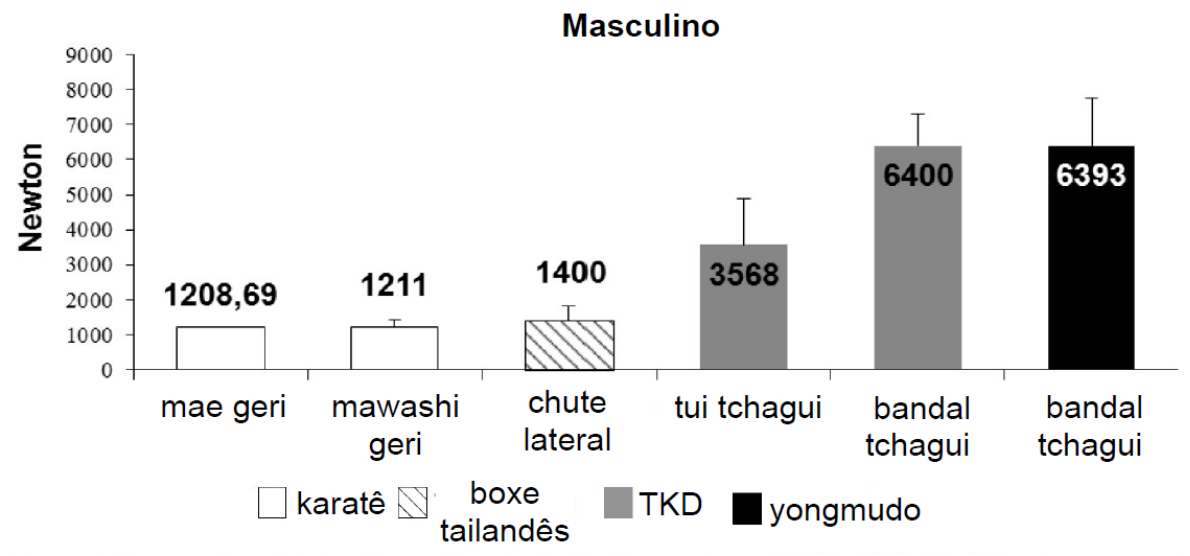

Figura 6. Impacto do chute masculino conforme a técnica. Fonte: ilustração elaborada pelo autor baseado nos dados de (Cynarski et al., 2018; Buśko et al., 2016; Buśko \& Nikolaidis, 2018; Estevan et al., 2011; Falco et al., 2009; Gavagan \& Sayers, 2017; O Sullivan et al., 2009). 
Os dados da figura 2B foram passados para impacto relativo. Então, o impacto relativo do soco do boxe masculino conforme a categoria por peso foi apresentado em ordem crescente na figura 7.

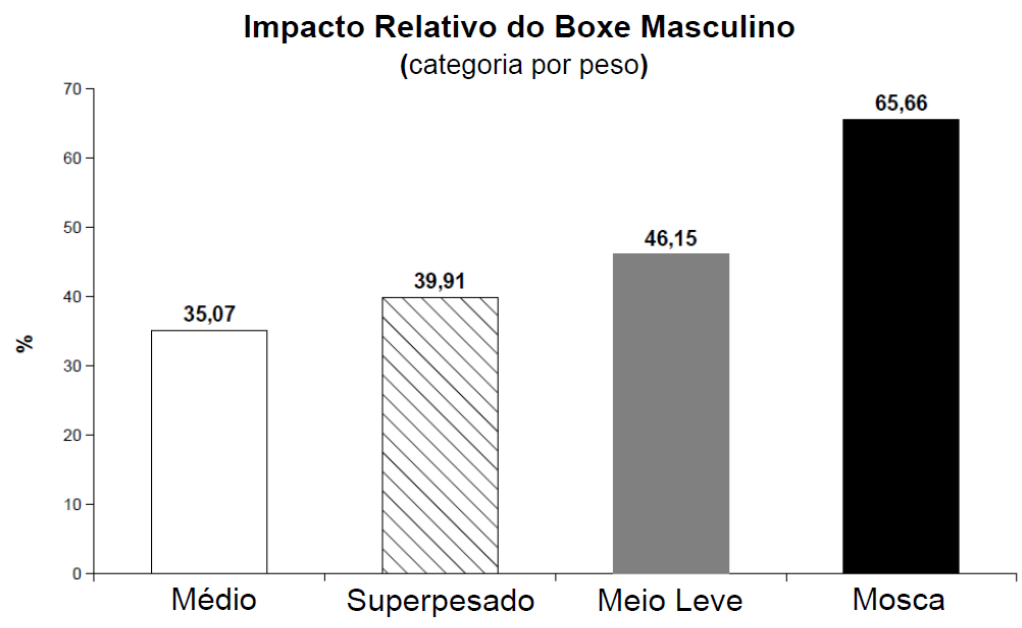

Figura 7. Impacto relativo (IR) do soco conforme a categoria por peso. Fonte: ilustração elaborada pelo autor através do cálculo do IR que usou os dados de Walilko et al. (2005).

Os dados da figura 3A e 3B foram passados para impacto relativo. Então, 7 tipos de soco com impacto relativo masculino estão na figura 8A e 3 tipos de soco do boxe com impacto relativo feminino estão na figura $8 \mathrm{~B}$, eles foram apresentados em ordem crescente.

A

Impacto Relativo do Soco Masculino

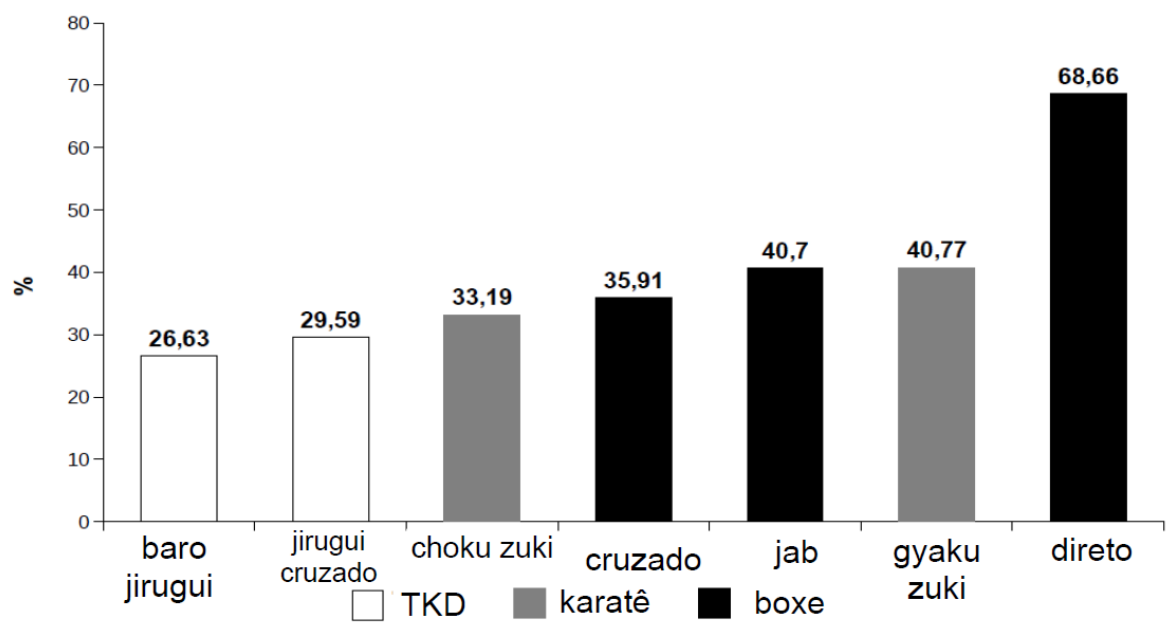




\section{B}

Impacto Relativo do Boxe Feminino

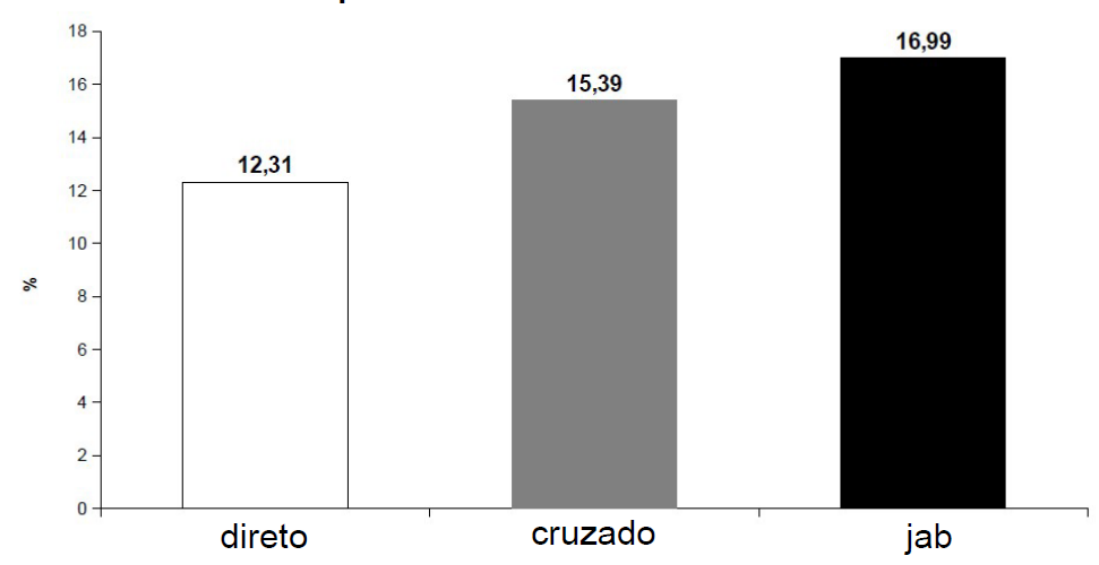

Figura 8. Impacto relativo (IR) do tipo de soco (A) masculino. (B) feminino do boxe. Fonte: ilustrações elaboradas pelo autor através do cálculo do IR que usou os dados de (Buśko et al., 2016; Buśko \& Nikolaidis, 2018; Cruz et al., 2014; Dunn et al., 2019; Gulledge \& Dapena, 2008; Loturco et al., 2016; Souza \& Marques, 2017; Todeschini Viero, 2012; Touguinho, 2014; Turner et al., 2011; Venkatraman \& Nasiriavanaki, 2019).

Os dados da figura 4 a 6 foram passados para impacto relativo. Então, os 3 tipos de chute com impacto relativo feminino estão na figura $9 \mathrm{~A}$ e os 6 tipos de chute com impacto relativo masculino estão na figura $9 \mathrm{~B}$, eles foram apresentados em ordem crescente.

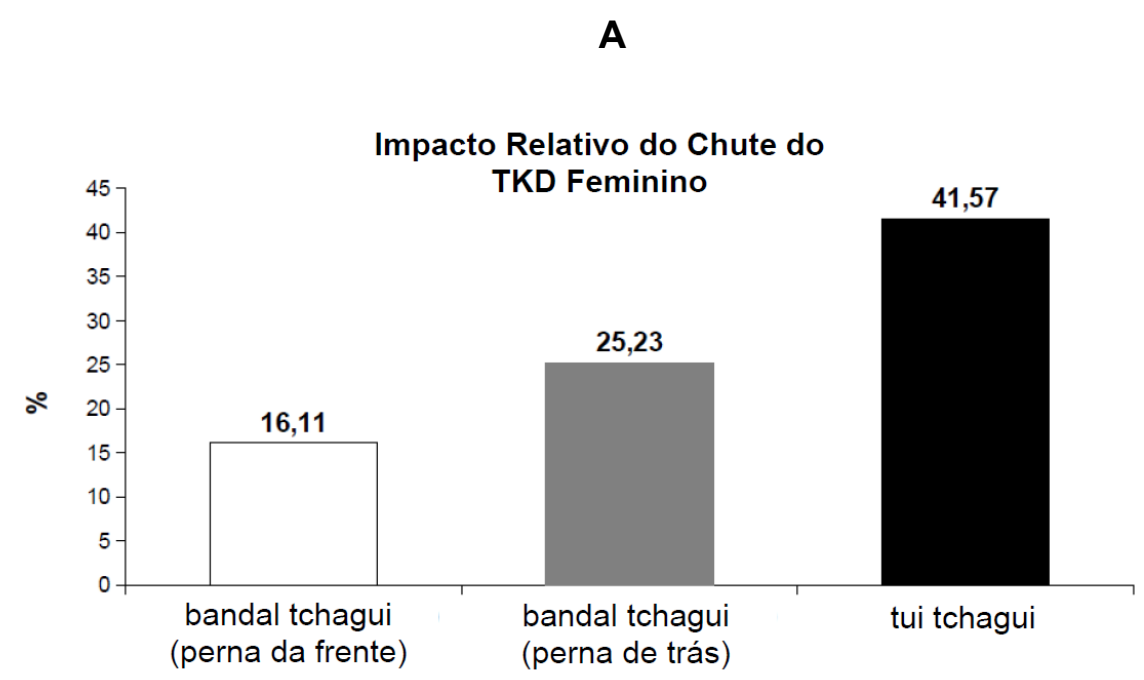




\section{B}

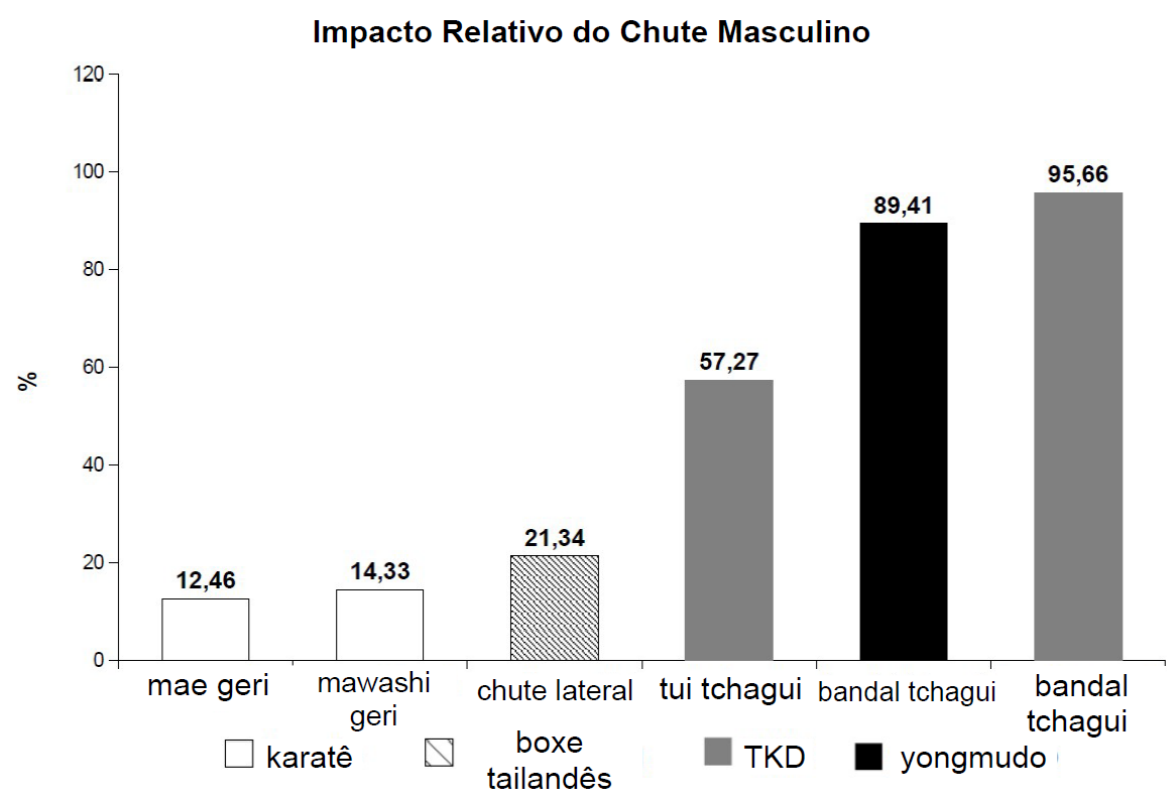

Figura 9. Impacto relativo (IR) do tipo de chute (A) masculino. (B) feminino. Fonte: ilustrações elaboradas pelo autor através do cálculo do IR que usou os dados de (Cynarski et al., 2018; Buśko et al., 2016; Buśko \& Nikolaidis, 2018; Estevan et al., 2011; Falco et al., 2009; Gavagan \& Sayers, 2017; O`Sullivan et al., 2009)

\section{DISCUSSÃO}

O mais alto impacto absoluto do soco foi do super pesado do boxe e esteve conforme a $2^{\mathrm{a}}$ Lei de Newton (Força = massa em kg. Aceleração em m/s =? N), mas a expectativa era de um menor impacto do soco do peso mosca, mas ele atingiu o $2^{\circ}$ melhor impacto do soco, seguido do peso meio leve e finalmente o peso médio - veja a figura 2B (Walilko et al., 2005). Entretanto, o peso mosca atingiu o $2^{\circ}$ melhor impacto absoluto do soco e $1^{\circ}$ valor do impacto relativo do soco. Então, essa categoria teve uma melhor performance, mas são necessários mais estudos para essas afirmações serem conclusivas.

O soco com rotação do tronco com a mão de trás da guarda teve um alto impacto, isso ocorreu no soco direto do boxe masculino (Turner et al., 2011) e no gyaku zuki do karatê shotokan (Todeschini Viero, 2012), ver figura 3A. Talvez o maior impacto do soco com rotação do tronco esteja relacionado com a maior velocidade desse ataque que outros tipos de soco. Outra hipótese do maior impacto do soco com rotação do tronco é por causa das características ofensivas das tarefas dessas lutas, elas usam mais soco no combate, os lutadores de boxe utilizam vários sacos de pancada para aumentar a força do soco e os 
karatecas utilizam soco no makiwara para ter um golpe mais potente. Entretanto, esses resultados merecem estudo para saber o motivo desse ocorrido. O maior impacto do soco feminino foi do jab (ver figura 3B) (Buśko et al., 2016; Loturco et al., 2016), mas razão disso não foi encontrado na literatura (Buśko, 2019; Markovic et al., 2016).

O impacto relativo do soco teve o mesmo resultado do impacto absoluto do soco - ver figura $\underline{3 A}$ e $\underline{8 A}$, o maior impacto foi do direto do boxe (Turner et al., 2011) e em segundo foi o gyaku zuki do karatê shotokan (Todeschini Viero, 2012). Esse resultado foi do lutador masculino. Outro similar resultado ocorreu no soco feminino do boxe, o impacto relativo teve o mesmo resultado do impacto absoluto, o mais alto impacto foi do jab, em segundo foi do cruzado e em terceiro foi o direto Buśko et al., $\underline{2016}$; Loturco et al., $\underline{2016}$ ) - ver figura $\underline{3 \mathrm{~B}}$ e $\underline{8 \mathrm{~B}}$.

O karatê e o TKD os atletas praticam o soco e/ou o chute com kiai para o ataque ter mais energia e consequentemente o resultado da força do soco e/ou do chute ser maior e proporcionar um mais elevado impacto dessa tarefa ofensiva (Nakayama, 2012; Ohnishi \& Onishi, 2008; Tanaka, 2001). Segundo Berti et al. (2019), o kiai o atleta pratica durante o ataque e na defesa com vocalização forte e profunda. Masic (1987) evidenciou que o gyaku zuki com kiai foi de 21,91 a 40,70 $\mathrm{N}$ e sem kiai foi de 22,60 a 35,07 N. Então, isso foi uma significativa limitação das pesquisas do impacto do soco e esse tema merece mais estudos.

O TKD feminino teve mais alto impacto do tui tchagui e em segundo ficou o bandal tchagui com a perna de trás da base (Buśko et al., 2016) - ver figura 4. Esses resultados foram maiores do que o chute lateral (yeop tchagui) do lutador de TKD masculino com $1120 \mathrm{~N}$ (Wasik, 2011). O impacto relativo apresentado na figura 9A do tui tchagui e do bandal tchagui foi maior do que (Buśko et al., 2016) o impacto relativo do chute lateral masculino de 14,93\% (Wasik, 2011). Portanto, o tipo de chute está relacionado com o impacto do chute, mas são necessárias mais pesquisas para essa informação ser conclusiva.

O bandal tchagui do TKD masculino com a perna de trás da base teve maior impacto do que com a perna da frente da base do mesmo chute $(p \leq 0.05)$ (Buśko et al., 2016; Buśko \& Nikolaidis, 2018) - ver tabela 2. O impacto relativo do bandal tchagui com a perna de trás foi de 30,92 a $33,26 \%$ e da perna da frente foi de 18 a 19,36\%. Esse resultado do impacto relativo foi similar ao do impacto absoluto - ver figura 2. Essa informação é importante durante o combate porque o lutador precisa priorizar o chute com a perna de trás da base no momento da disputa ou da luta real. A razão do maior impacto com a perna de trás da base é porque o chute é praticado com maior velocidade linear (Marques Junior, 2015). Outra informação importante sobre o bandal tchagui, esse chute tem mais impacto no tronco do que no rosto ( $p>0.05$ ) (O`Sullivan et al., 2009) - ver tabela 2. O mesmo foi evidenciado no impacto relativo (tronco com 89,41 a $95,66 \%$ e rosto com 76,57 a $81 \%$ ). O maior impacto no tronco é porque o chute é praticado com maior velocidade linear (Marques Junior, 2011; 2015). 
O técnico mensurar o impacto do ataque é uma importante tarefa para os esportes de combate que necessitam de bom soco e/ou chute durante a luta. Entretanto, nem sempre é possível do treinador ter equipamento altamente sofisticado para mensurar o impacto do ataque. Depois do técnico filmar o soco e/ou o chute no saco de pancada e/ou no makiwara, ele precisa usar o software Kinovea ${ }_{\circledast}$ (http://www.kinovea.org) para determinar a velocidade linear em metros por segundo $(\mathrm{m} / \mathrm{s})$ e o tempo do ataque segundos (s), para estabelecer o impacto do ataque com um cálculo matemático. Marques Junior e Silva Filho (2013) apresentam essa equação: Impacto do Ataque $=[$ (massa coporal total em kg. (velocidade linear máxima em $\left.\mathrm{m} / \mathrm{s})^{2}\right]$ : [2. tempo do ataque em $\left.\mathrm{s}\right]=$ ? $\mathrm{N}$.

A revisão sistemática teve limitações, foram encontrados poucos artigos ( $n=19)$ sobre o impacto do ataque. Então, os pesquisadores precisam de conduzir mais estudos sobre esse tema. Outra limitação dos estudos do impacto do soco e do chute é que utilizaram diferentes equipamentos e com diferentes padrões para coletar os dados do ataque do lutador. Então, os pesquisadores precisam padronizar o equipamento e a posição inicial para o atleta fazer o ataque durante a coleta dos dados.

\section{CONCLUSÃO}

A revisão sistemática detectou do soco e do chute o impacto absoluto e o impacto relativo. Entretanto, esse estudo não informou o impacto de todas as técnicas do soco e do chute das lutas estudadas (taekwondô, karatê, boxe, boxe tailandês e yongmudo). Então, os cientistas precisam praticar estudos sobre esse tema. Para futuras pesquisas sobre o impacto do soco e do chute dos esportes de combate é necessário investigar a relação entre o nível do impacto do ataque e a quantidade de vitórias na luta. Em conclusão, o impacto do soco e do chute dos esportes de combate é um importante conteúdo para o atleta conhecer o ataque mais eficaz.

\section{REFERÊNCIAS}

Atha, J., Yeadon, M. R., Sandover, J., \& Parsons, K.C. (1985). The damaging punch. British Medical Journal, 291(6511), 1756-1757. doi: https://dx.doi.org/10.1136\%2Fbmj.291.6511.1756

Berti, B., Momi, D., Sprugnoli, G., Neri, F., Bonifazi, M., Rossi, A., Muscettola, M. ... \& Rossi, S. (2019). Peculiarities of functional connectivity - including cross-modal patterns - in professional karate athletes: correlations with cognitive and motor performance. Hindawi Neural Plasticity, 2019, 1-15. doi: https://doi.org/10.1155/2019/6807978 
Bingul, B.M, Bulgan, C., Tore, O., Aydin, M., \& Bal, E. (2017). The effects of impact forces and kinematics of two different stances at strength punch techniques in boxing. Archives of Budo Science of Martial Arts and Extreme Sports, 13(1), 35-39. Recuperado de http://smaes.archbudo.com/view/abstract/id/11758

Bishop, S., Bounty, P., \& Devlin, M. (2013). Mixed martial arts: a comprehensive review. Journal of Sport and Human Performance, 1(1), 28-42. Recuperado de https://journals.tdl.org/ihp/index.php/JHP/article/view/jshp.0006.2013

Buśko, K. (2019). Biomechanical characteristics of amateur boxers. Archives of Budo, 15, 2331.

Recuperado

de

https://pdfs.semanticscholar.org/0dee/5222418c2d61146b24f6ebacb5b573195a4b.pdf

Buśko, K., Staniak, Z., Eckardi, M., Nikolaidis, P.T., Mazur-Różycka, J., Lach, P., Michalsk, R. ... Górski, M. (2016). Measuring the force of punches and kicks among combat sport athletes using a modified punching bag with an embedded accelerometer. Acta of Bioengineering and Biomechanics, 18(1), 47-54. doi: https://doi.org/10.5277/ABB$\underline{00304-2015-02}$

Buśko, K., \& Nikolaidis, P. (2018). Biomechanical characteristics of taekwondo athletes: kicks and punches vs. laboratory tests. Biomechanical Human Kinetics, 10(1), 81-88. doi: https://doi.org/10.1515/bhk-2018-0013

Cruz, F.S., Nagata, E.Y., \& Livramento, W.R. (2014). Análise da força de impacto e tempo de execução do soco gyaku zuki no karatê. Em XXIV Congresso Brasileiro de Engenharia Biomédica. Brasil. Recuperado de http://www.canal6.com.br/cbeb/2014/artigos/cbeb2014 submission 672.pdf

Cynarski, J., Wąsik, J., Szymczy, D., \& Vences de Brito, A. (2018). Changes in foot pressure on the ground during mae geri kekomi (front kick) in karate athletes - case study. Physical Education of Students, 22(1), 12-16. doi: https://doi.org/10.15561/20755279.2018.0102

Dunn, E., Humberstone, C., Iredale, K.F., \& Blazevich, A.J. (2019). A damaging punch: assessment and application of a method to quantify punch performance. Translational Sports Medicine, 2(3), 146-152. doi: https://doi.org/10.1002/tsm2.71

Estevan, I., Álvarez, O., Falco, C., Molina-García, J., \& Castillo, I. (2011). Impact force and time analysis influenced by execution distance in a roundhouse kick to the head in 
taekwondo. Journal of Strength and Conditioning Research, 25(10), 2851-2856. doi: https://doi.org/10.1519/JSC.0b013e318207ef72

Falco, C., Alvarez, O., Castillo, I., Estevan, I., Mortos, J., Mugarra, F., \& Iradi, A. (2009). Influence of the distance in a roundhouse kick's execution time and impact force in taekwondo. Journal of Biomechanics, 42(3), 242-248. doi: https://doi.org/10.1016/j.jbiomech.2008.10.041

Fernandes, F.M., Wichi, R.B., Silva, V.F., Ladeira, A.P.X., \& Ervilha, U.F. (2011). Biomechanical methods applied in martial arts studies. Journal Morphological Science, 28(3), 141-144. Recovered of https://pdfs.semanticscholar.org/7000/b999ac40d45350f0897371c84ec639b0d027.pdf

Franchini, E., \& Del Vecchio, F.B. (2011). Estudos em modalidades esportivas de combate: estado da arte. Revista Brasileira de Educação Física e Esporte, 25(Número especial), 67-81. Recuperado de https://www.revistas.usp.br/rbefe/article/download/16844/18557/0

Galna, A., Peter, A., Murphy, A.T., \& Morris, M.E. (2009). Obstacle crossing deficits in older adults: a systematic review. Gait Posture, 30(3), 270-275. doi: https://doi.org/10.1016/j.gaitpost.2009.05.022

Gavagan, C.L., \& Sayers, M.G.L. (2017). A biomechanical analysis of the roundhouse kicking technique of expert practitioners: a comparison between the martial arts disciplines of muay thai, karate, and taekwondo. Plos One, 12(8), e0182645. doi: https://doi.org/10.1371/journal.pone.0182645

Gulledge, J.K., \& Dapena, J. (2008). A comparison of the reverse and power punches in oriental martial arts. Journal of Sports Sciences, 26(2), 189-196. doi: https://doi.org/10.1080/02640410701429816

Halperin, I., Chapman, D.W., Martin, D.T., Lewthwaite, R., \& Wulf, G. (2017). Choice enhance punching performance of competitive kickboxers. Psychological Research, 81(5), 10511058. doi: https://doi.org/10.1007/s00426-016-0790-1

Hernández González, V., Sans-Rosell, N., Jové-Deltell, C., \& Reverter-Masia, J. (2018). Análisis bibliométrico de las tesis doctorales españolas en artes marciales y publicaciones científicas de sus autores. Movimento: Revista de Educação Física da UFRGS, 24(2), 367-382. doi: http://dx.doi.org/10.22456/1982-8918.77660 
Lee, B., \& McGill, S.M. (2014). Striking dynamics and kinetic properties of boxing and MMA gloves. Revista de Artes Marciales Asiáticas, 9(2), 106-115. doi: http://dx.doi.org/10.18002/rama.v9i2.1175

Loturco, I., Nakamura, F.Y., Artioli, G.G., Kobal, R., Kitamura, K., Abad, C.C. ... \& Franchini, E. (2016). Strength and power qualities are highly associated with punching impact in elite amateur boxers. Journal Strength and Conditioning Research, 30(1), 109-116. Recuperado de http://www.yakademia.hu/s and p.pdf

Markovic, P., Suzovic, D., Kasum, G., \& Jaric, S. (2016). Effects of training against elastic resistance on jab punch performance in elite junior athletes. Kinesiology, 48(1), 79-86. doi: https://doi.org/10.26582/k.48.1.8

Marques Junior, N.K. (2011). Karatê shotokan: biomecânica dos golpes do kumitê de competição. EFDeportes.com, 16(158), 1-28. Recuperado de https://www.efdeportes.com/efd158/karate-biomecanica-dos-golpes-do-kumite.htm

Marques Junior, N.K. (2015). Velocity of the offensive technique of the karate for the mixed martial arts fighter: a systematic review. Revista Observatorio del Deporte, 1(4), 29-62. Recuperado de http://www.revistaobservatoriodeldeporte.cl/gallery/2\%20oficial\%20articulo\%20vol\%201 \%20num\%204\%202015\%20rev\%20odep.pdf

Marques Junior, N.K., \& Silva Filho, J.N. (2013). Treino de força para o karateca do estilo shotokan especialista no kumite. Revista Brasileira de Prescrição e Fisiologia do Exercício, 7(41), 506-533. Recuperado de http://www.rbpfex.com.br/index.php/rbpfex/article/view/585

Masic, Z. (1987). Kiai and mechanical characteristics of some karate punches. Em $V$ International Symposium on Biomechanics. (pp. 167-173). Recuperado de https://ojs.ub.uni-konstanz.de/cpa/article/view/2318

Moher, D., Liberati, A., Tetzlaff, J., \& Altman, D. (2009). Preferred reporting items for systematic reviews and meta-analyses: the PRISMA statement. PLOS Medicine, 6(7), e1000097. doi: https://doi.org/10.1371/journal.pmed.1000097

Nakayama, M. (2012). O melhor do karatê 5 - heian e tekki (8ª ed). São Paulo: Cultrix.

Ohnishi, S.T., \& Ohnishi, T. (2009). Philosophy, psychology, physics and practice of ki. Evidence-Based Complementary and Alternative Medicine, 6(2), 175-183. Recovered of https://www.hindawi.com/journals/ecam/2009/814972/ 
O`Sullivan, D., Chung, C., Lee, K., Kim, E., Kang, S., Kim, T., \& Shim, I. (2009). Measurement and comparison of taekwondo and yongmudo turning kick impact force for two target heights. Journal of Sports Science and Medicine, 8(CSSI III), 13-16. Recuperado de https://www.ncbi.nlm.nih.gov/pmc/articles/PMC3879643/

Pinto Neto, O., Silva, J.H., Marzullo, A.C.M., Bolander, R.P., \& Bir, C.A. (2013). The effect of hand dominance on martial arts strikes. Human Movement Science, 31(4), 824-833. doi: https://dx.doi.org/10.1016\%2Fi.humov.2011.07.016

Sklyar, M.S. (2014). Correlation analysis of indicators of physical and technical preparedness karate school age. Pedagogics, psychology, 14, 56-62. doi: https://doi.org/10.6084/m9.figshare.950956

Souza, V.A, \& Marques, A.M. (2017). Relationship between age and expertise with the maximum impact force of a reverse punch by Shotokan karate athletes. Archives of Budo, 13, 243-254. Recuperado de http://archbudo.com/view/abstract/id/11561

Souza Junior, T.P., Ide, B.N., Sasaki, J.E., Lima, R.F., Abad, C.C.C., Leite, R.D., Barros, M.P., \& Utter, A.C. (2015). Mixed martial arts: history, physiology and training aspects. Open Sports Science Journal, 13(8), 1-7. doi: http://dx.doi.org/10.2174/1875399X01508010001

Tanaka, M. (2001). Karate-dô: perfecting kumite. Loewestr: Gotzelmann.

Touguinho, H. (2014). Análise biomecânica do soco direto de lutadores de artes marciais mistas (dissertação de mestrado). Universidade Camilo Castelo Branco, São José dos Campos, Instituto de Engenharia Biomédica, Brasil. Recuperado de https://sucupira.capes.gov.br/sucupira/public/consultas/coleta/trabalhoConclusao/viewTr abalhoConclusao.jsf?popup=true\&id trabalho $=2214053$

Turner, A., Baker, E., \& Miller, S. (2011). Increasing the impact force of the rear hand punch. Strength and Conditioning Journal, 33(6), 2-9. doi: https://doi.org/10.1519/SSC.0b013e318232fdcb

Venkatraman, J., \& Nasiriavanaki, M. (2019). Biomechanics of kumite style gyaku tsuki in karate. Biomedical Journal of Scientific \& Technical Research, 14(3), 10656-10662. doi: https://doi.org/10.26717/BJSTR.2019.14.002550

Todeschini Viero, F. (2012). Análise cinética do soco no karatê em postura natural e avançada (Dissertação de Mestrado). Universidade Do Estado De Santa Catarina, Florianópolis, 
Brasil.

Recuperado

de

http://sistemabu.udesc.br/pergamumweb/vinculos/00006d/00006d5a.pdf

Wasik, J. (2011). Kinematic analysis of the side kick in taekwondo. Acta of Bioengineering and Biomechanics, 13(4), 71-75. Recuperado de http://www.actabio.pwr.wroc.pl/Nol13No4/9.pdf

Walilko, T.J., Viano, D.C., \& Bir, C.A. (2005). Biomechanics of the head for Olympic boxer punches to the face. British Journal of Sports Medicine, 39(10), 710-719. doi: http://dx.doi.org/10.1136/bjsm.2004.014126 